\title{
Chronotherapy for rheumatoid arthritis: current perspectives
}

This article was published in the following Dove Press journal:

ChronoPhysiology and Therapy

3 August 2016

Number of times this article has been viewed

\section{Hideto To \\ Department of Medical Pharmaceutics, Graduate School of Medicine and Pharmaceutical Sciences for Research, University of Toyama, Toyama, Japan}

\begin{abstract}
Rheumatoid arthritis (RA) is an autoimmune disorder of unknown etiology. Morning stiffness, a characteristic feature of RA, shows a 24-hour rhythm. Cytokines, which are considered to play an important role in the pathogenesis of RA, also exhibit a 24-hour rhythm, with a peak in the early morning. These rhythms have been attributed to the endogenous hormone balance and changes in expression levels of clock-related genes. Chronotherapy based on the 24-hour rhythm of RA has been performed using glucocorticoids and disease-modifying antirheumatic drugs. In a previous study, it was reported that modified-release prednisone tablets were administered to patients with RA at night, which demonstrated that the severity of morning stiffness was markedly less than that in patients receiving the standard treatment. Methotrexate (MTX) is the most frequently used RA drug worldwide. In a basic study, cytokines and inflammatory responses in RA model animals showed 24-hour rhythms, based on which MTX was administered and exerted dosing time-dependent antirheumatic effects. Plasma C-reactive protein and cytokine levels also exhibit 24-hour rhythms in patients with RA, with peaks occurring in the early morning. MTX has been shown to markedly inhibit the exacerbation of arthritis in patients with RA when it is administered as inflammatory responses and tumor necrosis factor- $\alpha$ levels begin to increase. Tacrolimus (TAC) is an immunosuppressive agent that is administered to patients who undergo organ transplants. Since one of the mechanisms of action of TAC is the inhibition of inflammatory cytokine production, it is used as an RA therapeutic drug. When TAC was previously administered in the early light or early dark phase to RA model animals, the group treated in the early light phase had notably inhibited increase in arthritis scores compared with that in the early dark phase. The selection of an optimal dosing time associated with 24-hour rhythms in RA symptoms may lead to more effective and safer treatments for RA using glucocorticoids and disease-modifying antirheumatic drugs.
\end{abstract}

Keywords: methotrexate, steroid, circadian rhythm, cytokines

\section{Chronobiology of rheumatoid arthritis}

Rheumatoid arthritis (RA) is a systemic disease in which arthritis is the most important and common symptom. Extra-articular manifestations are often present when the disease is aggressive. Marked joint inflammation may lead to arthritic pain and the destruction of joints. Joint deformities and a loss of function ultimately occur with the progression of RA. ${ }^{1,2}$ Although many requirements must be met in order to establish a diagnosis of RA, morning stiffness is a characteristic feature and occurs in many patients. Pain, functional disability, and stiffness show 24-hour rhythms in many patients with RA, with a peak in the early morning. ${ }^{3,4}$ The 24-hour rhythms in pain and stiffness have been attributed to daily variations
Department of Medical Pharmaceutics, Graduate School of Medicine and Pharmaceutical Sciences for Research, University of Toyama, 2630 Sugitani, Toyama 930-0194, Japan $\mathrm{Tel} / \mathrm{fax}+817641588 \mathrm{II}$

Email hide-to@umin.net

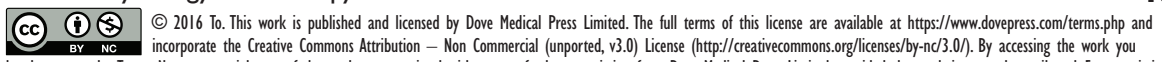
hereby accept the Terms. Non-conmercial uses of the work are permitted without any further permisision from Dove Medical Press Limited, provided the work is properly atributed. For permision for commercial use of this work, please see paragraphs 4.2 and 5 of our Terms (htpps//www.dovepress.com/terms.php). 
in local and systemic inflammatory responses. A previous study reported that plasma C-reactive protein (CRP) levels, an indicator of inflammatory responses, showed a 24-hour rhythm in patients with RA with a peak in the early morning and trough in the evening, which matched the 24-hour rhythms in pain and stiffness. ${ }^{5}$ Proinflammatory cytokines, such as tumor necrosis factor- $\alpha$ (TNF- $\alpha$ ) and interleukin (IL)-6, are secreted from activated monocytes and macrophages and have been shown to increase the levels of CRP in hepatocytes. Clear 24-hour rhythms have been observed in the blood concentrations of these cytokines, with higher levels being reported in the early morning in patients with RA. ${ }^{6}$ However, no significant 24-hour rhythm in plasma IL-6 levels has been detected in healthy humans. Therefore, 24-hour rhythms in cytokines play central roles in the pathogenesis of RA.

Besides cytokines, the circadian rhythm and levels of melatonin (MLT) and glucocorticoids and a clock gene, Cryptochrome (Cry), may have effects on the 24-hour rhythm of RA symptoms. Arthritis scores and serum TNF- $\alpha$ concentrations were previously shown to be higher in $\mathrm{Cryl}^{-/} \mathrm{Cr} y 2^{-}$mice than in the wild-type mice when both mice were inoculated anti-type II collagen monoclonal antibody in order to induce RA-like arthritis. ${ }^{7}$ Thus, Cry may play an important role in the induction of RA. MLT is known to enhance the expression of inflammatory cytokines, ${ }^{8}$ while glucocorticoids suppress the expression of cytokines such as IL-1, IL-6, and TNF- $\alpha$. Both MLT and glucocorticoids have been shown to exhibit clear 24-hour rhythms. ${ }^{9,10}$ Previous studies showed that MLT levels peaked 2 hours earlier and the peak level of cortisol was lower in patients with RA than in healthy controls. ${ }^{11,12}$ Cytokine overexpression in patients with RA may be caused by a change in the balance between the circadian rhythms of MLT and corticosterone.

The mechanisms responsible for the 24-hour rhythms in RA symptoms have not yet been elucidated. However, the changes in concentrations of endogenous hormones and expression levels of clock-related genes compared with healthy controls appear to play important roles in the development of RA.

\section{Chronotherapy for RA}

Although RA symptoms exhibit 24-hour rhythms, therapy based on these rhythms (chronotherapy) has not yet been developed. Chronopharmacology and chronotherapies that have been examined in basic and clinical studies are discussed in the following sections.

\section{Glucocorticoids}

Glucocorticoids have been used in RA therapy due to their anti-inflammatory activities against symptoms such as joint stiffness and joint pain. Cortisol is an endogenous glucocorticoid, which reaches the peak in the morning in humans. Steroids are generally administered in the morning in order to prevent disturbances in the circadian rhythms of endogenous glucocorticoids. Two previous studies attempted to clarify the dosing time dependency of glucocorticoids, which were administered in the morning or night according to the 24-hour rhythms of endogenous glucocorticoids and RA symptoms. ${ }^{13,14}$ The findings revealed that the duration of morning stiffness and blood IL-6 levels were markedly shorter and lower, respectively, in the night dosing group than in the morning dosing group. However, chronotherapy using glucocorticoids has not been widely performed in patients with RA. This may be explained by concerns about dosing in the middle of the night and abnormal secretion of endogenous glucocorticoids.

In recent years, chronotherapy using glucocorticoids has achieved important outcomes. A modified-release formulation of prednisone has been developed, which releases the agent $\sim 4$ hours after its ingestion; therefore, patients with RA do not need to take the drug during the night (2-3 am) to get the antiinflammatory effects induced by glucocorticoids. When patients with RA were randomly given a modified-release tablet at bedtime or an immediate-release prednisone tablet in the morning, relative changes in the duration of joint morning stiffness were significantly higher with the modified-release tablet than with the immediate-release tablet. ${ }^{15}$ Moreover, a large number of studies have demonstrated that the safety and effectiveness of these tablets in that chronotherapy do not reduce hypothalamicpituitary-adrenal axis function or the secretion of endogenous glucocorticoids and are effective for the patients with RA who do not respond to immediate-release prednisone. ${ }^{16,17}$ Therefore, chronotherapy using modified-release prednisone tablets may be expected to become a useful RA therapy.

\section{Disease-modifying antirheumatic drugs} Methotrexate

Methotrexate (MTX) decreases the production of cytokines by suppressing the proliferation of lymphocytes ${ }^{18}$ and TNF- $\alpha$ transcriptional activity, ${ }^{19}$ inhibits joint inflammation, ${ }^{20,21}$ and induces a high American College of Rheumatology improvement response rate ${ }^{22}$ in patients with RA. Many factors must be considered prior to the administration of MTX in order to prevent the development of adverse effects such as myelosuppression and interstitial pneumonitis. Inflammatory responses 
have been implicated in morning stiffness, a well-known characteristic of RA. However, MTX has not been examined using a dosing schedule based on the 24-hour rhythms.

Collagen-induced arthritis (CIA) represents a true autoimmune reaction against major joint components associated with class II major histocompatibility complex genes and pannus formation. The CIA model is similar to RA in terms of pathology, immunology, and genetics. ${ }^{23,24}$ In a basic study using rats with CIA, blood concentrations of CRP showed a significant 24-hour rhythm, with higher concentrations being observed in the late dark phase to the early light phase (Figure 1). ${ }^{25}$ A previous study administered MTX once daily at Zeitgeber time (ZT) 10 or ZT22 and found that the arthritis score was significantly lower in the ZT22 group than in the control and ZT10 groups in rats with CIA (Figure 1). ${ }^{26}$ On day 22, the inhibition rate of the arthritis score in the ZT22 group was approximately fivefold higher than that in the ZT10 group. Moreover, plasma TNF- $\alpha$ levels were found to be 3.03- to 5.39-fold higher in mice with CIA than in normal mice at all sampling times after the onset of RA. These levels showed a significant 24-hour rhythm with a peak at ZT2 and trough at ZT14. When MTX was intraperitoneally injected at ZT10 or ZT22, arthritis scores were significantly lower in the ZT22 group than in the control and ZT10 groups. On the other hand, the arthritis scores of the ZT10 group did not

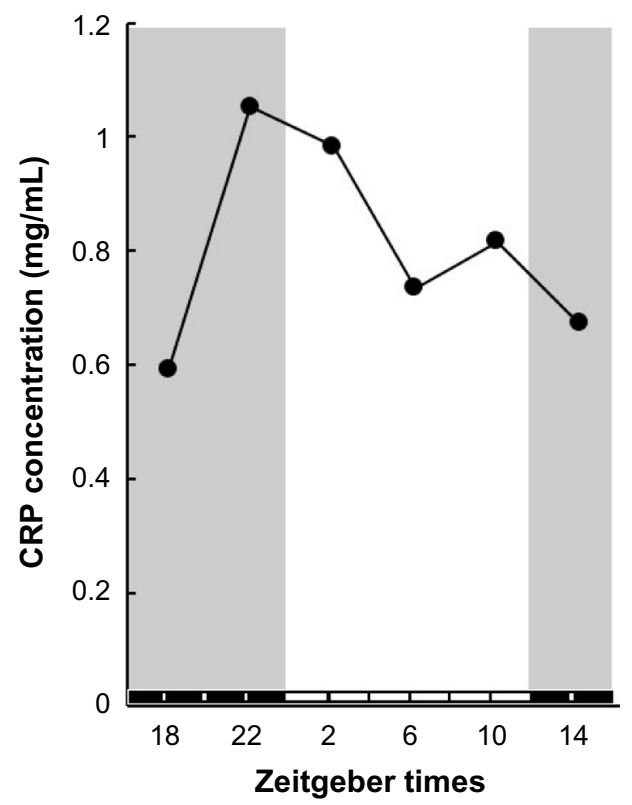

significantly differ from those of the control group for the entire study period in mice. ${ }^{26}$

In our previous studies using CIA model, MTX dosing groups showed no significant adverse effects such as myelosuppression. MTX is an anticancer drug, the administration of which is often associated with adverse effects such as myelosuppression. However, the dose of MTX in RA therapy is $1 / 10$ of that in cancer chemotherapy. Thus, MTX may reduce plasma TNF- $\alpha$ levels by suppressing transcriptional activity rather than suppressing lymphocyte proliferation.

In basic studies, 24-hour rhythms have been reported in inflammatory responses and cytokine levels after the onset of RA. Better antirheumatic effects may be obtained when MTX is administered at specific times in synchronization with circadian rhythms. Therefore, the selection of an optimal dosing time based on circadian rhythms in RA symptoms may lead to more effective MTX treatments for RA.

MTX is mainly administered three times a week (day 1: after breakfast and supper and day 2: after breakfast only) to Japanese patients with RA regardless of the total MTX dose per week. Based on our findings in animal studies and the circadian TNF- $\alpha$ rhythms of the patients with RA, we performed a prospective, single-arm study, in which we switched from the standard MTX schedule (MTX is administered three times a week) to a chronotherapy schedule

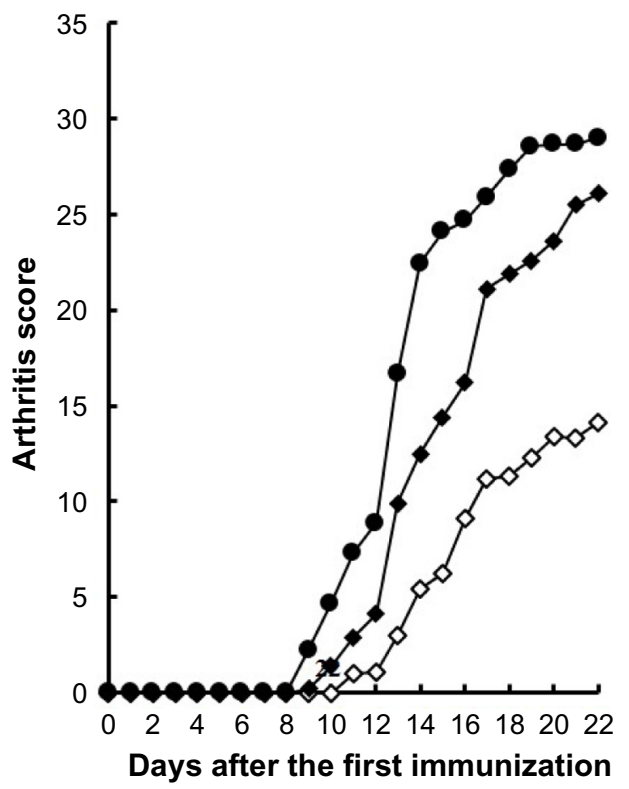

Figure I The 24-hour rhythm in CRP levels and the dosing time dependency of MTX in rats with CIA.

Notes: Regarding CRP levels, each value represents the mean of six rats with CIA. A significant 24-hour rhythm was observed in CRP concentrations. In order to estimate antirheumatic effects, MTX $(0.1 \mathrm{mg} / \mathrm{kg}$ ) was injected po at ZT22 (open diamond) or ZTI0 (closed diamond) every day for 3 weeks in rats with CIA ( $\mathrm{n}=10$ ). The control group (closed circle) was administered sodium bicarbonate (7\%). Each value represents the mean. The arthritis score was significantly lower in the ZT22 group than in the control and ZTI0 groups. A previously described arthritis scoring system was used that evaluated individual joints and weighted the arthritis severity by joint size, as follows: (a) for the interphalangeal joints, each of the 4 lateral digits in the hind legs was scored as 0 or I ( $0=$ no arthritis and I=arthritis present); and (b) for the ankle and midfoot joints, each was scored on a scale of $0-4(0=$ normal, I= minimal swelling, $2=$ moderate swelling, $3=$ severe swelling, and $4=$ severe swelling and non-weight bearing). Abbreviations: CIA, collagen-induced arthritis; CRP, C-reactive protein; MTX, methotrexate; po, postoperatively; ZT, Zeitgeber time. 
(MTX is administered once a day before bedtime), and the dose and number of doses per week were the same as the standard MTX schedule for each Japanese patient with RA. ${ }^{27}$ Table 1 shows the time courses of tender and swollen joint counts (28 joints), CRP levels, a global self-assessment of disease activity, and Disease Activity Score (DAS) 28-CRP as an indicator of disease activity in this study. ${ }^{28}$ Twenty-two patients with RA aged between 41 years and 78 years were enrolled. Seventeen (77\%) patients received chronotherapy with MTX for the 3-month study period. Five patients dropped out due to retraction of acceptance $(n=1)$, out of inclusion criteria just before study $(\mathrm{n}=1)$, lack of monitoring data $(n=1)$, and receive an operation without disease caused by RA $(n=2)$. The tender joint count decreased slightly after chronotherapy. On the other hand, the swollen joint count decreased markedly from 1.18 at the baseline ( 0 month) to 0.29 at 3 months. The CRP level continued to improve throughout the 3-month study period and had improved by $64.2 \%$ after chronotherapy from that at the baseline. Visual Analog Scale (VAS) was not significantly affected, despite patients recognizing the change in the MTX administration method. DAS28 was markedly improved after chronotherapy from that at the baseline. The therapeutic effects of using DAS28 are estimated by the European League Against Rheumatism (EULAR) response rates. The EULAR response rates have been classified as follows: good responders are patients displaying an improvement of $>1.2$ and a present score of $\leq 2.7$; moderate responders are patients displaying an improvement of $>0.6$ and $\leq 1.2$ and a present score of $\leq 4.1$ or an improvement of $>1.2$ and a present score of $>4.1$; and nonresponders are any patients displaying an improvement of $\leq 0.6$ or patients displaying an improvement of $>0.6$ and $\leq 1.2$ and a present score of $>4.1 .^{29}$ On the basis of the EULAR definitions of treatment responses according to DAS28 at 3 months, seven (41.2\%) patients achieved a moderate response, and four (23.5\%) attained clinical remission (Figure 2) ${ }^{27}$ Four out of five ( $80 \%$ ) males and three out of $12(25 \%)$ females achieved a moderate response, and

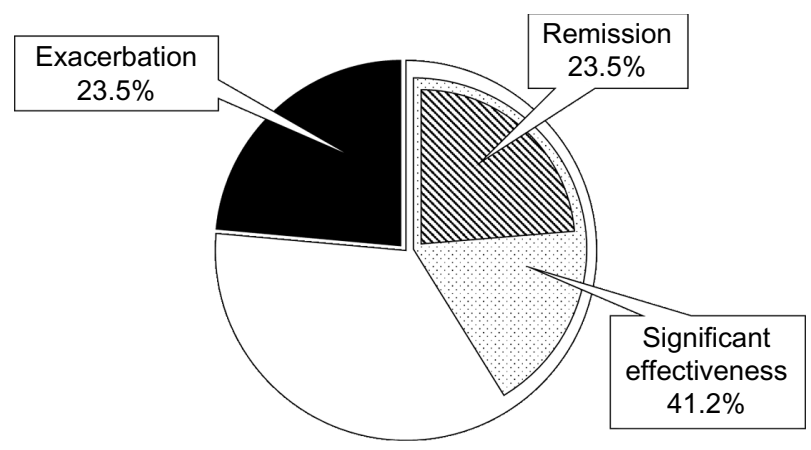

Figure 2 Therapeutic estimation based on EULAR definitions for chronotherapy with MTX in patients with RA.

Abbreviations: EULAR, European League Against Rheumatism; MTX, methotrexate; RA, rheumatoid arthritis.

males obtained greater therapeutic effects than females. Although most patients had mild leukopenia, the incidence of leukopenia higher than grade 1 increased from $11.8 \%$ to $23.5 \%$ throughout the study. Moreover, no severe adverse effects occurred in 17 patients. These findings demonstrated that chronotherapy with MTX is safe and markedly improves disease activity and the functional capacity of patients with RA. We are now conducting a double-blind, randomized, controlled trial of chronotherapy with MTX.

Chronotherapy with MTX is also being carefully examined in a clinical study, and selecting an optimal dosing time in consideration of 24-hour rhythms in RA symptoms is expected to lead to more effective MTX therapies for RA.

\section{Tacrolimus}

Tacrolimus (TAC) is used as prophylaxis for organ rejection in patients undergoing transplants such as kidney, liver, or heart. Its immunosuppressive effects inhibit the activation of calcineurin, a phosphatase. ${ }^{30,31}$ These effects are exerted by inhibiting the mRNA transcription of cytokine production such as that of IL-2, which is required for the activation of T-cells. ${ }^{32}$ TAC has been used in Japan as an RA therapeutic drug because it inhibits the production of inflammatory cytokines. $^{33,34}$

Table I Single-arm study of methotrexate chronotherapy in Japanese patients with RA

\begin{tabular}{lllll}
\hline & \multicolumn{4}{l}{ Time after start of chronotherapy (month) } \\
\cline { 2 - 5 } & $\mathbf{0}$ & $\mathbf{I}$ & $\mathbf{2}$ & $\mathbf{3}$ \\
\hline Number of tender joints & $10.4 \pm 7.6$ & $8.76 \pm 8.07$ & $8.76 \pm 8.20$ & $8.88 \pm 7.78$ \\
Number of swollen joints & $1.18 \pm 1.5 \mathrm{I}$ & $0.18 \pm 0.73$ & $0.41 \pm 1.46$ & $0.29 \pm 0.99$ \\
CRP levels (mg/dL) & $0.66 \pm 1.29$ & $0.67 \pm 1 . I 1$ & $0.54 \pm 0.94$ & $0.43 \pm 0.71$ \\
Patient's global assessment of their disease activity (VAS) & $35.5 \pm 25.7$ & $32.9 \pm 23.4$ & $32.1 \pm 21.9$ & $32.9 \pm 22.0$ \\
DAS28-CRP & $3.83 \pm 0.62$ & $3.37 \pm 1.12$ & $3.33 \pm 1.08$ & $3.31 \pm 1.04$ \\
\hline
\end{tabular}

Note: Each value represents the mean \pm SD $(n=17)$. Disease Activity Score $(D A S) 28-C R P$ values were calculated as follows: $D A S 28(C R P)=0.56 \times \sqrt{ }(T) C 28)+0.28 \times$ $\sqrt{ }(\mathrm{SJC} 28)+0.014 \times \mathrm{GH}+0.36 \times \ln (\mathrm{CRP}+\mathrm{I})+0.96$ where $\mathrm{TJC}=$ tender joint count, SJC $=$ swollen joint count, and $\mathrm{GH}=$ general health.

Abbreviations: CRP, C-reactive protein; RA, rheumatoid arthritis; DAS, Disease Activity Score; VAS, Visual Analog Scale. 
The adverse effects of TAC depend on dosing times in rats, and previous studies have demonstrated that groups treated in the active phase were at higher risk of adverse effects than those treated in the inactive phase. ${ }^{35,36}$ The reason for this has been attributed to the maximum blood concentration $\left(\mathrm{C}_{\max }\right)$ being markedly higher in the active phase than in the inactive phase in mice. ${ }^{37}$ Previous studies reported that the dosing time dependency of pharmacokinetics in addition to $\mathrm{C}_{\max }$ and the area under the plasma concentration-time curve were greater in patients treated during the daytime than in those treated at night. ${ }^{38,39}$ These findings are consistent with dosing time-dependent changes in adverse effects.

TAC is administered once a day after dinner to patients with RA in order to prevent adverse effects. However, it has not yet been determined whether TAC needs to be administered once daily after dinner in order to achieve better antirheumatic effects in patients with RA. Figure 3 shows 24-hour rhythms in TNF- $\alpha$ levels before and after the onset of RA, and dosing time differences in the arthritis score for TAC in mice with CIA. ${ }^{40}$ Plasma TNF- $\alpha$ concentrations were found to be markedly higher in mice with CIA than in normal mice. Mice with CIA showed a significant 24-hour rhythm in TNF- $\alpha$ levels, with higher levels in the light phase and lower levels in the dark phase. This type of change was also observed for IL-6 and serum amyloid A (SAA). The 24-hour rhythms observed in these mice correspond to those in patients with RA. Since 24-hour rhythms in cytokines and SAA levels peaked during the light phase and were the lowest during the dark phase, TAC was administered at ZT2 or ZT14 to mice with CIA. Although increases in the arthritis score were not inhibited in the ZT14 group, the suppression of arthritis was markedly stronger in the ZT2 group than in the control group (Figure 3). The dosing time when TAC showed greater antirheumatic effects in mice was similar to that of MTX in our previous studies on animals, ${ }^{22,27}$ and both drugs had stronger inhibitory effects on arthritis during the early morning when cytokine levels increased. The selection of an optimal dosing time according to the 24-hour cycling of inflammatory cytokines may contribute to improvements in the RA therapeutic effects of TAC.

\section{Conclusion}

RA symptoms such as morning stiffness exhibit 24-hour rhythms. Chronotherapies using glucocorticoids and diseasemodifying antirheumatic drugs that target these rhythms have been examined and achieved more beneficial outcomes than existing methods. We are currently performing basic and clinical studies to confirm the utility of chronotherapy involving various antirheumatic drugs, with the aim of developing safer and more effective RA therapies.

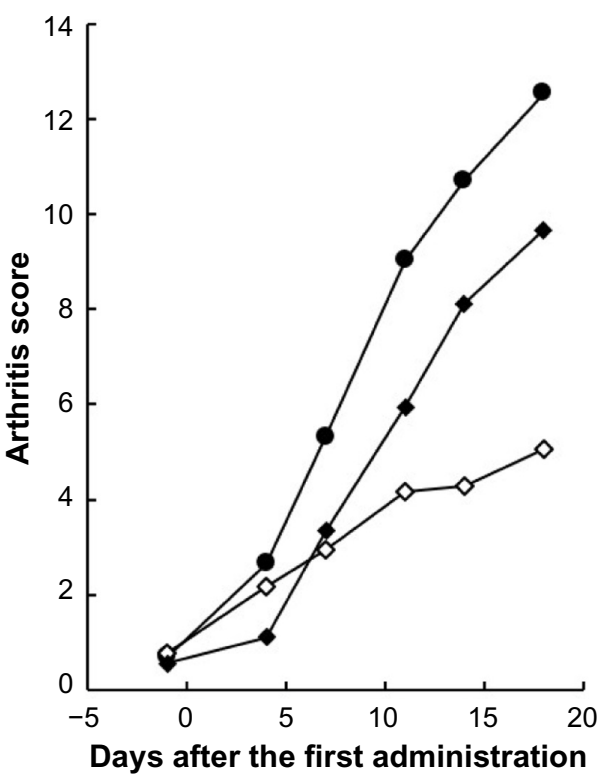

Figure 3 The 24-hour rhythm in TNF- $\alpha$ levels and the dosing time dependency of TAC in mice with CIA.

Notes: Regarding TNF- $\alpha$ levels, each value represents the mean of five or six normal mice (closed triangle) and eleven to 16 mice with CIA (closed circle). A significant 24-hour rhythm in TNF- $\alpha$ levels was observed in mice with CIA. In order to estimate antirheumatic effects, TAC (4 mg/kg) was ip administered once a day at ZT2 (open diamonds) or ZTI4 (closed diamonds) for 3 weeks ( $n=18$, respectively). Saline was administered to the control group (closed circle; $n=35$ ). Each value represents the mean. The arthritis score was markedly lower in the ZT2 group than in the control and ZTI4 groups. The mice were considered to have arthritis when significant changes in redness and/or swelling were noted in their digits or in other parts of their paws. Each inflamed toe counted as I point. Arthritis was graded on a scale of $0-5$ for each wrist/ ankle: $0=$ no changes; $I=$ slight erythema of the limbs, $2=$ minimal swelling, $3=$ moderate swelling and erythema of the limbs, $4=$ marked swelling and erythema of the limbs, $5=$ maximal swelling and redness of the limbs and ankylosis.

Abbreviations: CIA, collagen-induced arthritis; ip, intraperitoneally, TAC, tacrolimus; TNF- $\alpha$, tumor necrosis factor- $\alpha$; ZT, Zeitgeber time. 


\section{Disclosure}

The authors report no conflicts of interest in this work.

\section{References}

1. Gabriel SE. The epidemiology of rheumatoid arthritis. Rheum Dis Clin North Am. 2001;27(2):269-281.

2. Harris ED Jr. Rheumatoid arthritis. Pathophysiology and implications for therapy. N Engl J Med. 1990;322(18):1277-1289.

3. Bellamy N, Sothern RB, Campbell J, Buchanan WW. Circadian rhythm in pain, stiffness, and manual dexterity in rheumatoid arthritis: relation between discomfort and disability. Ann Rheum Dis. 1991;50(4):243-248.

4. Kowanko IC, Knapp MS, Pownall R, Swannell AJ. Domiciliary selfmeasurement in the rheumatoid arthritis and the demonstration of circadian rhythmicity. Ann Rheum Dis. 1982;41(5):453-455.

5. Herold M, Günther R. Circadian rhythm of C-reactive protein in patients with rheumatoid arthritis. Prog Clin Biol Res. 1987;227B:271-279.

6. Crofford LJ, Kalogeras KT, Mastorakos G, et al. Circadian relationships between interleukin (IL)-6 and hypothalamic-pituitary-adrenal axis hormones: failure of IL-6 to cause sustained hypercortisolism in patients with early untreated rheumatoid arthritis. J Clin Endocrinol Metab. 1997;82(4):1279-1283.

7. Hashiramoto A, Yamane T, Tsumiyama K, et al. Mammalian clock gene cryptochrome regulates arthritis via proinflammatory cytokine TNF-alpha. J Immunol. 2010;184(3):1560-1565.

8. Garcia-Mauriño S, Gonzalez-Haba MG, Calvo JR, et al. Melatonin enhances IL-2, IL-6, and IFN-gamma production by human circulating CD4+ cells: a possible nuclear receptor-mediated mechanism involving $\mathrm{T}$ helper type 1 lymphocytes and monocytes. J Immunol. 1997;159(2):574-581.

9. Gavrila A, Peng CK, Chan JL, Mietus JE, Goldberger AL, Mantzoros CS. Diurnal and ultradian dynamics of serum adiponectin in healthy men: comparison with leptin, circulating soluble leptin receptor, and cortisol patterns. J Clin Endocrinol Metab. 2003;88(6):2838-2843.

10. Kennaway DJ, Voultsios A. Circadian rhythm of free melatonin in human plasma. J Clin Endocrinol Metab. 1998;83(3):1013-1015.

11. Sulli A, Maestroni GJ, Villaggio B, et al. Melatonin serum levels in rheumatoid arthritis. Ann NY Acad Sci. 2002;966:276-283.

12. Neeck G, Federlin K, Graef V, Rusch D, Schmidt KL. Adrenal secretion of cortisol in patients with rheumatoid arthritis. J Rheumatol. 1990;17(1):24-29.

13. De Silva M, Binder A, Hazleman BL. The timing of prednisolone dosage and its effect on morning stiffness in rheumatoid arthritis. Ann Rheum Dis. 1984;43(6):790-793.

14. Arvidson NG, Gudbjörnsson B, Elfman L, Rydén AC, Tötterman TH, Hällgren R. Circadian rhythm of serum interleukin-6 in rheumatoid arthritis. Ann Rheum Dis. 1994;53(8):521-524.

15. Buttgereit F, Doering G, Schaeffler A, et al. Efficacy of modified-release versus standard prednisone to reduce duration of morning stiffness of the joints in rheumatoid arthritis (CAPRA-1): a double-blind, randomised controlled trial. Lancet. 2008;371(9608):205-214.

16. Alten R, Döring G, Cutolo M, et al. Hypothalamus-pituitary-adrenal axis function in patients with rheumatoid arthritis treated with nighttimerelease prednisone. J Rheumatol. 2010;37(10):2025-2031.

17. Alten R, Holt R, Grahn A, et al. Morning stiffness response with delayed-release prednisone after ineffective course of immediate-release prednisone. Scand J Rheumatol. 2015;44(5):354-358.

18. Williams AS, Topley N, Dojcinov S, Richards PJ, Williams BD. Amelioration of rat antigen-induced arthritis by liposomally conjugated methotrexate is accompanied by down-regulation of cytokine mRNA expression. Rheumatology. 2001;40(4):375-383.

19. Becker C, Barbulescu K, Hildner K, Meyer zum Büschenfelde KH, Neurath MF. Activation and methotrexate-mediated suppression of the TNF $\alpha$ promoter in T cells and macrophages. Ann NY Acad Sci. 1998;859:311-314.

20. Kremer JM, Phelps CT. Long-term prospective study of the use of methotrexate in the treatment of rheumatoid arthritis. Update after a mean of 90 months. Arthritis Rheum. 1992;35(2):138-145.
21. Weinblatt ME, Weissman BN, Holdsworth DE, et al. Long-term prospective study of methotrexate in the treatment of rheumatoid arthritis. 84-month update. Arthritis Rheum. 1992;35(2):129-137.

22. Cohen S, Cannon GW, Schiff M, et al. Two-year, blinded, randomized, controlled trial of treatment of active rheumatoid arthritis with leflunomide compared with methotrexate. Arthritis Rheum. 2001;44(9):1984-1992.

23. Wooley PH, Luthra HS, Krco CJ, Stuart JM, David CS. Type II collagen-induced arthritis in mice. II. Passive transfer and suppression by intravenous injection of anti-type II collagen antibody or free native type II collagen. Arthritis Rheum. 1984;27(9):1010-1017.

24. Holmdahl R, Andersson ME, Goldschmidt TJ, et al. Collagen induced arthritis as an experimental model for rheumatoid arthritis. Immunogenetics, pathogenesis and autoimmunity. APMIS. 1989;97(7):575-584.

25. Kanasaki Y, Tomonari M, Sasaki H, To H. Chronopharmacology of mizoribine in collagen-induced arthritis rats. J Pharmacol Sci. 2012;120(2):112-120.

26. To H, Irie S, Tomonari M, Watanabe Y, Kitahara T, Sasaki H. Therapeutic index of methotrexate depends on circadian cycling of tumour necrosis factor-alpha in collagen-induced arthritic rats and mice. J Pharm Pharmacol. 2009;61(10):1333-1338.

27. To H, Yoshimatsu H, Tomonari M, et al. Methotrexate chronotherapy is effective against rheumatoid arthritis. Chronobiol Int. 2011;28(3):267-274.

28. van Gestel AM, Prevoo ML, van't Hof MA, van Rijswijk MH, van de Putte LB, van Riel PL. Development and validation of the European League Against Rheumatism response criteria for rheumatoid arthritis. Comparison with the preliminary American College of Rheumatology and the World Health Organization/International League Against Rheumatism Criteria. Arthritis Rheum. 1996;39(1):34-40.

29. Fransen J, van Riel PL. The disease activity score and the EULAR response criteria. Clin Exp Rheumatol. 2005;23(5 suppl 39):S93-S99.

30. Dumont FJ. FK506, An immunosuppressant targeting calcineurin function. Curr Med Chem. 2000;7(7):731-748.

31. Miyatake S, Kaminuma O. Inhibitors of NFAT-calcineurin pathway. Nihon Rinsho. 2005;63(9):1633-1639.

32. Kino T, Hatanaka H, Miyata S, et al. FK506, a novel immunosuppressant isolated from a Streptomyces. II. Immunosuppressive effect of FK506 in vitro. J Antibiot. 1987;40(9):1256-1265.

33. Sakuma S, Kato Y, Nishigaki F, et al. FK506 potently inhibits $\mathrm{T}$ cell activation induced TNF-alpha and IL-1beta production in vitro by human peripheral blood mononuclear cells. $\mathrm{Br} J$ Pharmacol. 2000;130(7):1655-1663.

34. Sakuma S, Kato Y, Nishigaki F, et al. Effects of FK506 and other immunosuppressive anti-rheumatic agents on $\mathrm{T}$ cell activation mediated IL-6 and IgM production in vitro. Int Immunopharmacol. 2001;1(4):749-757.

35. Fujimura A, Ebihara A. Administration time-dependent toxicity of a new immunosuppressive agent, tacrolimus (FK 506). Life Sci. 1994;55(7):485-490.

36. Uchida H, Kobayashi E, Ogino Y, et al. Chronopharmacology of tacrolimus in rats: toxicity and efficacy in a mouse-to-rat intestinal transplant model and its pharmacokinetic profile. Transplant Proc. 1999;31(7):2751-2753.

37. Fujimura A, Shiga T, Ohashi K, Ebihara A. Chronopharmacokinetic study of a new immunosuppressive agent, FK 506, in mice. Jpn J Pharmacol. 1993;61(2):137-139.

38. Min DI, Chen HY, Fabrega A, et al. Circadian variation of tacrolimus disposition in liver allograft recipients. Transplantation. 1996;62(8):1190-1192.

39. Baraldo M, Furlanut M. Chronopharmacokinetics of cyclosporine and tacrolimus. Clin Pharmacokinet. 2006;45(8):775-788.

40. Obayashi K, Tomonari M, Yoshimatsu H, et al. Dosing time-dependency of the arthritis-inhibiting effect of tacrolimus in mice. J Pharmacol Sci. 2011;116(3):264-273. 


\section{Publish your work in this journal}

ChronoPhysiology and Therapy is an international, peer-reviewed, open access journal focusing on research into the cyclic variations and rhythmicity in physiological processes in the body and the research and development and optimal timing of administration of therapeutic targets to achieve improved outcomes and quality of life for the patient. The

Submit your manuscript here: http://www.dovepress.com/chronophysiology-and-therapy-journal

\section{Dovepress}

manuscript management system is completely online and includes a very quick and fair peer-review system. Visit http://www.dovepress.com/ testimonials.php to read real quotes from published authors. 